Notre Dame Journal of Formal Logic

Volume 47, Number 3, 2006

\title{
Invariant Version of Cardinality Quantifiers in Superstable Theories
}

\author{
Alexander Berenstein and Ziv Shami
}

\begin{abstract}
We generalize Shelah's analysis of cardinality quantifiers from Chapter V of Classification Theory and the Number of Nonisomorphic Models for a superstable theory. We start with a set of bounds for the cardinality of each formula in some general invariant family of formulas in a superstable theory (in Classification Theory, a uniform family of formulas is considered) and find a set of derived bounds for all formulas. The set of derived bounds is sharp: up to a technical restriction, every model that satisfies the original bounds has a sufficiently saturated elementary extension that satisfies the original bounds and such that for each formula the set of its realizations in the extension has arbitrarily large cardinality below the corresponding derived bound of the formula.
\end{abstract}

\section{Introduction}

Cardinality quantifiers in stable theories were studied by Shelah in Chapter V, Section 6 of [3]. Roughly, Shelah's setting is as follows. Let $T$ be a stable theory in a language $L$ and $\mathbb{C}$ a large saturated model of $T$. Let $W$ be a set of triples $\left(\varphi(\bar{x}, \bar{y}), \psi(\bar{y}), \chi^{+}\right)$, where $\varphi(\bar{x}, \bar{y}), \psi(\bar{y}) \in L$, and $\chi \geq|T|$ is a cardinal such that $T$ is stable in $\chi$ (in general, $\chi$ satisfies more technical assumptions). The collection $W$ is called a set of demands. A model $M=T$ is said to be $W$-good if, for any tuple $\bar{a}$ of $M$ and triple $\left(\varphi(\bar{x}, \bar{y}), \psi(\bar{y}), \chi^{+}\right) \in W$, if $M \models \psi(\bar{a})$, then $|\varphi(M, \bar{a})|<\chi^{+}$. In this case the model $M$ is said to satisfy the demands $W$.

Given $W$, Shelah introduced, for each $\varphi(\bar{x}, \bar{a}) \in L(\mathbb{C})$, a cardinal $C(\varphi(\bar{x}, \bar{a}))$ called a derived bound. On the one hand, the derived bounds must be proper bounds, in the sense that any $a$-model $M$ that is $W$-good also satisfies $|\varphi(M, \bar{a})|<C(\varphi(\bar{x}, \bar{a}))$. Secondly, they are minimal in the following sense. Let $h: \mathrm{Car} \rightarrow$ Car be a function such that $h(\lambda)<\lambda$ for all $\lambda$. An $a$-model $M$ is said to be $h$-bounded if for any

Received April 1, 2005; accepted March 6, 2006; printed November 14, 2006

2000 Mathematics Subject Classification: Primary, 03C45, 03C50

Keywords: cardinality quantifiers, superstable theories

(C)2006 University of Notre Dame 
formula $\varphi(x, \bar{a}) \in L(M)$ we have $|\varphi(M, \bar{a})| \geq h(C(\varphi(x, \bar{a})))$. Shelah proved the following.

Theorem 1.1 (Theorem V.6.7 in [3]) Let $W$ be a set of demands. Let $M$ be an $a$-model which is $W$-good. Let $h: \mathrm{Car} \rightarrow \mathrm{Car}$ be nondecreasing with $h(\lambda)<\lambda$ for all $\lambda$. Then there is an a-model $N \succeq M$ such that $N$ is $W$-good and $h$-bounded.

The condition that $h$ is nondecreasing is not stated in [3], but was meant to be added by Shelah (as pointed out to the second author in discussions with him). Note that the set of demands $W$ in Shelah's setting is required to satisfy a continuity condition; that is, for any given $\varphi(x, y) \in L$ if we require that the cardinality of $\varphi(x, a)$ is less than $\chi^{+}$in a model, then we must require that the cardinality of $\varphi\left(x, a^{\prime}\right)$ is less than $\chi^{+}$for all $a^{\prime}$ in some formula of $t p(a)$.

The goal in this paper is to generalize the theorem stated above to the context of a general Aut $(\mathbb{C})$-invariant set of demands $W$ on a general Aut($(\mathbb{C})$-invariant collection of formulas. That is, we remove the continuity condition and consider instead a set $W$ of triples $\left(\varphi(\bar{x}, \bar{y}), p(\bar{y}), \chi^{+}\right)$, where $p(\bar{y})$ is a complete type. We succeed in generalizing the theorem when the theory is superstable. To prove our results we introduce a new set of derived bounds. In fact, for his result (Theorem 1.1) Shelah had to prove the type-definability of the relations $C(\varphi(\bar{x} ; \bar{b})) \geq \kappa$, for any fixed $L$ formula $\varphi$ and cardinal $\kappa$. The type-definability of these relations cannot be proved in our setting even for any proper set of derived bounds $C$; this can be shown by a simple example. In our work, in order to refine the derived bounds for formulas, we define new derived bounds for all small types.

This paper is organized as follows. In Section 2 we recall mostly basic results about stable theories proved in [3]. We also need two technical results from [3], namely, Facts 2.7 and 2.8. The basic facts that we list also appear in [1] and [2]. In Section 3 we introduce the derived bounds and prove the main result.

\section{Preliminaries}

Throughout this paper $T$ is assumed to be a stable theory and $\mathbb{C}$ a large saturated model of $T$. We use capital letters such as $A, B, C, \ldots$ for subsets of $\mathbb{C}$ and whenever we say that $A$ is a set, we mean that $|A|<\|\mathbb{C}\|$. We will denote partial types by $p(\bar{x})$; if the variables of the partial type $p(\bar{x})$ are clear from context we may just write $p$. We will assume the reader is familiar with basic results in stability theory that appear in [1], [2], and [3]. We will also use the following definitions and facts.

Definition 2.1 ([3], Definition 1.1, Chap. IV) $\quad$ Let $\lambda \geq \kappa_{r}(T)$. By an $F_{\lambda}^{a}$-saturated model $M$ we mean a model $M \models T$ such that for any $A \subset M$ of cardinality less than $\lambda$, every type over acl ${ }^{\mathrm{eq}}(A)$ is realized in $M$. An $a$-model is an $F_{\kappa_{r}(T)}^{a}$-saturated model.

If $B$ is a set and $\lambda \geq \kappa_{r}(T)$ is a regular cardinal, there is a prime $F_{\lambda}^{a}$-saturated model over $B$ that is unique up to isomorphism over $B$. We denote it by $\operatorname{Pr}_{F_{\lambda}^{a}}(B)$. If $\lambda=\kappa_{r}(T)$, we denote the prime $a$-model as $\operatorname{Pr}_{a}(B)$.

Fact 2.2 ([3], Theorem 2.8, Chap. I) $\quad$ Assume $T$ is $\lambda$-stable and let $I, A$ be sets such that $|I|>\lambda \geq|A|$. Then there is $I^{\prime} \subset I$ of cardinality greater than $\lambda$, which is an indiscernible sequence over $A$. 
Fact 2.3 (Folklore) Let $A, B, I$ be sets and assume that $I$ is an indiscernible sequence over $A$. Then there is $I^{\prime} \subset I$ of cardinality $\leq|B|+\kappa(T)$ such that $I \backslash I^{\prime}$ is an indiscernible sequence over $A \cup B \cup I^{\prime}$.

Fact 2.4 ([2], Lemma 4.5.9, Chap. 1) Let $M$ be a model, $\bar{a} \in M, p \in S(M)$, and $\varphi(\bar{x}, \bar{a})$ a formula in $p$. Then $p\left\lceil_{\varphi(M, \bar{a})} \bigcup \bar{a} \models p\right.$.

Fact 2.5 ([2], Lemma 4.1.2, Chap. 1) Assume $T$ is $\lambda$-stable. Then $T$ has a $\lambda$ saturated model of power $\lambda$.

Fact 2.6 ([3], Theorem 4.9, Chap. IV) $\quad$ Let $A$ be a set and suppose $\operatorname{cf}(\lambda) \geq \kappa_{r}(T)$. Let $M=\operatorname{Pr}_{F_{\lambda}^{a}}(A)$ be an $F_{\lambda}^{a}$-prime model over $A$. Then every $A$-indiscernible sequence $I \subseteq M$ is of size $\leq \lambda$.

We also need some more technical results.

Fact 2.7 ([3], Lemma 6.2, Chap. V) Let $p \in S(A)$ be a stationary type and $\psi(\bar{x}, \bar{b}) \in L(\mathbb{C})$. Then the following conditions are equivalent (and we denote them by $p \not \perp \psi)$.

1. For every $\kappa \geq|A|^{+}+\kappa_{r}(T)$ and $F_{\kappa}^{a}$-saturated model $M$, if $\bar{b} \cup A \subseteq M$ then $\operatorname{dim}(p, M)<|\psi(M, \bar{b})|^{+}+\aleph_{0}$.

2. For every $\kappa \geq \kappa_{r}(T)$ there is an $F_{\kappa}^{a}$-saturated model (eq. for all $F_{\kappa}^{a}$-saturated model) $M$ such that $\bar{b} \cup A \subseteq M$ and there is a nonforking extension $\operatorname{tp}(\bar{c} / M)$ of $p$ such that any $F_{\kappa}^{\bar{a}}$-prime model $N=\operatorname{Pr}_{F_{\kappa}^{a}}(M \cup \bar{c})$ satisfies $\psi(N, \bar{b}) \supseteqq \psi(M, \bar{b})$.

3. For every infinite indiscernible sequence $I$ based on $p, I$ is not indiscernible over $\psi(\mathbb{C}, \bar{b}) \cup \bar{b}$.

4. Not every infinite indiscernible sequence $J \subseteq \psi(\mathbb{C}, \bar{b})$ is orthogonal to $p$.

Fact 2.8 ([3], Lemma 6.8(iii), Chap. V) $\quad$ Let $M \models T, \varphi(\bar{x}, \bar{a}), \psi(\bar{y}, \bar{b}) \in L(M)$, $\bar{d}_{1} \models \varphi(\bar{x}, \bar{a}), \bar{d}_{2} \models \psi(\bar{y}, \bar{b})$ and assume that $\bar{d}_{1} \bigotimes_{M} \bar{d}_{2}$. Let $\theta\left(\bar{x}, \bar{m}, \bar{d}_{2}\right)$ be a formula in $\operatorname{tp}\left(\bar{d}_{1} / M \cup \bar{d}_{2}\right)$ that forks over $M$ and such that $\theta\left(\bar{x}, \bar{m}, \bar{d}_{2}\right) \vdash \varphi(\bar{x}, \bar{a})$. Then there exists an equivalence relation $E \in L(\varphi(M, \bar{a}) \cup \bar{a})$ such that $|\{c / E: c \in M\}| \leq|\psi(M, \bar{b})|$ and $\bar{d}_{1}^{\prime} / E \notin M^{\mathrm{eq}}$ for all $\bar{d}_{1}^{\prime} \models \theta\left(\bar{x}, \bar{m}, \bar{d}_{2}\right)$.

\section{The Main Theorem}

In this section, $T$ is assumed to be a stable theory.

\section{Notation 3.1}

1. Let $W=\left\{\left(\varphi_{i}(\bar{x}, \bar{y}), p_{i}(\bar{y}), \chi_{i}^{+}\right) \mid i \in I\right\}$, where $\varphi_{i}(\bar{x}, \bar{y}) \in L(\varnothing), p_{i} \in S(\varnothing)$, $\chi_{i} \geq|T|, T$ is stable in $\chi_{i}$, and $I$ is an index set.

2. We write $\models_{W} \exists^{<\chi} \bar{x} \varphi(\bar{x}, \bar{a})$ if there is $(\varphi(\bar{x}, \bar{y}), p(\bar{y}), \chi) \in W$ such that $\models p(\bar{a})$.

3. Let $\operatorname{Car}(W)=\{\chi \mid(\varphi(\bar{x}, \bar{y}), p(\bar{y}), \chi) \in W$ for some $\varphi(\bar{x}, \bar{y}), p(\bar{y})\}$.

Definition 3.2 Let $\varphi(\bar{x}, \bar{y}) \in L$, let $\chi$ be a cardinal, and let $p \in S(\varnothing)$. We say that $M$ is $\varphi$, $p$-good in $\chi$ if whenever $\bar{b} \in M$ realizes $p$, then $|\varphi(M, \bar{b})| \leq \chi$. We say $M \models T$ is $W$-good if for all $\bar{a} \in \mathbb{C}$, whenever $\models_{W} \exists^{<\chi} \bar{x} \varphi(\bar{x}, \bar{a})$ we have $|\varphi(M, \bar{a})|<\chi$.

We will define a set of derived bounds on partial types, that is, a set of consequences of $\models_{W} \exists^{<\chi} \bar{x} \varphi(\bar{x}, \bar{a})$. For doing this, we need to restrict ourselves to partial types over small sets. 
Definition 3.3 A partial type $p$ over a set $A$ is said to be small if $|A| \leq|T|$. A cardinal $\lambda$ is said to be a stability bound if $\lambda=\chi^{+}$and $T$ is stable in $\chi$.

Definition 3.4 Let $C:$ \{partial small types $\rightarrow$ Car. We say that $C$ is a derived set of bounds if it satisfies the following properties:

1. If $\models_{W} \exists^{<\chi} \bar{x} \varphi(\bar{x}, \bar{a})$ then $C(\varphi(\bar{x}, \bar{a})) \leq \chi$. If $\varphi(\bar{x}, \bar{a})$ is algebraic of multiplicity $n$, then $C(\varphi(\bar{x}, \bar{a})) \leq n+1$.

2. If $F$ is a definable function defined on $\varphi_{1}(\bar{x}, \bar{a})$, then $C\left(F\left(\varphi_{1}(\bar{x}, \bar{a})\right)\right) \leq$ $C\left(\varphi_{1}(\bar{x}, \bar{a})\right)$.

3. Let $E$ be a definable equivalence relation, $p(\bar{x})$ a small type, and $\lambda$ a regular cardinal. If $C(\{\bar{x} / E \mid$ all $\bar{x}\}) \leq \lambda$ and $C(p(\bar{x}) \wedge E(\bar{x}, \bar{c})) \leq \lambda$ for every $\bar{c}$, then $C(p(\bar{x})) \leq \lambda$.

4. $C\left(p_{1}\right) \leq C\left(p_{2}\right)$ for small $p_{1}$ and $p_{2}$ such that $p_{1} \vdash p_{2}$.

5. Let $\lambda$ be a stability bound and let $p$ be a small partial type over a set $A$ (so $|A| \leq|T|)$ such that all its completions $p^{+}$over $A$ satisfy $C\left(p^{+}\right) \leq \lambda$. Then $C(p) \leq \lambda$.

For any two derived sets of bound $C, C^{\prime}$, write $C^{\prime} \leq C$ if for all partial small types $p, C^{\prime}(p) \leq C(p)$. To build a derived set of bounds $C$ maximal with respect to $\leq$ we proceed as follows. We define a decreasing sequence $\left(C_{\alpha}: \alpha \leq \lambda\right)$ where $\lambda$ is large enough. Define $C_{0}(p)$ to be $\infty$ for each small partial type $p$. Then, for every $\alpha<\lambda$, $C_{\alpha}$ is obtained from the $C_{\beta}$ s, for $\beta<\alpha$, by first taking the minimum of its previous values (i.e., at each $p$ we take the minimum of $C_{\beta}(p)$ for $\beta<\alpha$ ) and then applying one of the rules (1) to (5). We continue this process until each of the $C_{\alpha}(p)$ does not decrease. Clearly, $C_{\lambda}$ is a derived set of bounds and it is maximal. From now on we fix $C$ to be a maximal derived set of bounds.

Remark 3.5 Let $M \models T$ be $W$-good. Then for every formula $\varphi(\bar{x}, \bar{a}) \in L(M)$ we have that $C(\varphi(\bar{x}, \bar{a}))>|\varphi(M, \bar{a})|$.

Lemma 3.6 Assume $T$ is stable in $\chi, M \models T$ is $\omega$-saturated, $\varphi(\bar{x}, \bar{y}) \in L$, and $p \in S(\varnothing)$. If for all $\bar{a} \in M$ realizing $p$ we have $|\varphi(M, \bar{a})| \leq \chi$, then for all $\bar{b} \in \mathbb{C}$ realizing $p$ we also have $|\varphi(\mathbb{C}, \bar{b}) \cap M| \leq \chi$.

Proof Let $\bar{b} \in \mathbb{C}$ be a realization of $p$ and assume, as a way to a contradiction, that $\chi<|\varphi(\mathbb{C}, \bar{b}) \cap M|$. Since $T$ is stable in $\chi$, by Fact 2.2 there is an indiscernible sequence $I \subseteq \varphi(\mathbb{C}, \bar{b}) \cap M$ of cardinality greater than $\chi$. Since $T$ is stable, there is $n=n(\varphi(x, \bar{y}))<\omega$ such that for every indiscernible sequence $I^{\prime}$ and for every $\bar{b}^{\prime}$, $\left|\left\{\bar{e}^{\prime} \in I^{\prime}: \varphi\left(\bar{e}^{\prime}, \bar{b}^{\prime}\right)\right\}\right|<n \vee \mid\left\{\bar{e}^{\prime} \in I^{\prime}: \neg \varphi\left(\bar{e}^{\prime}, \bar{b}^{\prime}\right) \mid<n\right.$.

Choose $\bar{e}_{1}, \ldots, \bar{e}_{n} \in I$ different from each other; then

$$
\mathbb{C} \models \bigwedge_{i=1}^{n} \varphi\left(\bar{e}_{i}, \bar{b}\right) \wedge p(\bar{b}) .
$$

By $\omega$-saturation of $M$, there is $\bar{b}^{\prime} \in M$ such that

$$
M \models \bigwedge_{i=1}^{n} \varphi\left(\bar{e}_{i}, \bar{b}^{\prime}\right) \wedge p\left(\bar{b}^{\prime}\right) .
$$

From the definition of $n, n \leq\left|\left\{\bar{e} \in I: \varphi\left(\bar{e}, \bar{b}^{\prime}\right)\right\}\right|$ implies $\left|\left\{\bar{e} \in I: \varphi\left(\bar{e}, \bar{b}^{\prime}\right)\right\}\right|>\chi$, which contradicts the assumption. 
Proposition 3.7 Assume $T$ is $\chi$-stable; let $\varphi(\bar{x}, \bar{y}) \in L$ and $p \in S(\varnothing)$. Let $\delta$ be a cardinal such that $\operatorname{cf}(\delta) \leq \chi$ and let $\left(M_{i}: i<\delta\right)$ be a sequence of models such that $M_{i} \preceq M_{j}$ for $i<j<\delta$. Assume that each $M_{i}$ is an a-model which is $\varphi$, $p$-good in $\chi$. Then for any set $A$ such that $|A| \leq \chi, \operatorname{Pr}_{a}\left(\cup_{i<\delta} M_{i} \cup A\right)$ is $\varphi$, p-good in $\chi$.

Proof Since $T$ is $\chi$-stable, $\kappa_{r}(T) \leq \chi$. Let $M=\cup_{i<\delta} M_{i}$ and let $M_{A}=\operatorname{Pr}_{a}(M \cup A)$. Let $\bar{b} \in M_{A}$ be a realization of $p$. To prove the proposition we will show

(a) there exists a model $M \cup \bar{b} \subseteq M^{*} \preceq \mathbb{C}$ such that $\left|\varphi\left(M^{*}, \bar{b}\right)\right| \leq \chi$;

(b) if there is $M^{*}$ as in (a) then $\left|\varphi\left(M_{A}, \bar{b}\right)\right| \leq \chi$.

We start with the second part.

(b) Suppose there is $M^{*} \preceq \mathbb{C}$ such that $M \cup \bar{b} \subseteq M^{*}$ and $\left|\varphi\left(M^{*}, \bar{b}\right)\right| \leq \chi$. Assume, in order to get a contradiction, that $\chi^{+} \leq\left|\varphi\left(M_{A}, \bar{b}\right)\right|$. Since $T$ is stable in $\chi$, by Fact 2.2 there exists $I \subseteq \varphi\left(M_{A}, \bar{b}\right)$ of cardinality $\chi^{+}$which is indiscernible over $\varphi\left(M^{*}, \bar{b}\right) \cup \bar{b}$. Thus all elements in $I$ have the same strong type over $\varphi\left(M^{*}, \bar{b}\right) \cup \bar{b}$. By Fact 2.4, $I \bigsqcup_{\varphi\left(M^{*}, \bar{b}\right) \cup \bar{b}} M^{*}$, so the sequence $I$ is indiscernible over $M^{*}$. In particular, $I$ is indiscernible over $M$. Since $|A| \leq \chi, T$ is stable in $\chi$, and $|I|=\chi^{+}$, by Fact 2.3 there is $I^{\prime} \subset I$ of cardinality $\chi^{+}$such that $I^{\prime}$ is indiscernible over $M \cup A$. Since $I^{\prime} \subset M_{A}$ and $M_{A}$ is $F_{\kappa_{r}(T)}^{a}$-prime over $M \cup A$, this contradicts Fact 2.6.

(a) We now prove that there exists $M \cup \bar{b} \subseteq M^{*} \preceq \mathbb{C}$ that satisfies $\left|\varphi\left(M^{*}, \bar{b}\right)\right| \leq \chi$. Our strategy to build $M^{*}$ will be the following: first, we build a chain of length less that $\chi^{+}$of models that contain $\bar{b}$ and that collect all elements in $M$ that depend on $\varphi(\bar{x}, \bar{b})$ over the previous elements in the chain. $M^{*}$ will be the $a$-prime model containing the supremum of the chain and $M$.

Notice that $T$ is stable in $\chi, M_{j}$ is $\omega$-saturated, and $\varphi, p$-good in $\chi$, so Lemma 3.6 implies $\chi \geq\left|\varphi(\mathbb{C}, \bar{b}) \cap M_{j}\right|$. Since $\operatorname{cf}(\delta) \leq \chi$, we also get $\chi \geq|\varphi(\mathbb{C}, \bar{b}) \cap M|$. Since $T$ is stable in $\chi$, by Fact 2.5 there is $N_{0}$ a saturated model of cardinality $\chi$ such that $(\varphi(\mathbb{C}, \bar{b}) \cap M) \cup \bar{b} \subseteq N_{0}$.

We define by induction a sequence of pairs $\left(\left(N_{i}, \bar{a}_{i}\right): i<\chi^{+}\right)$, where $N_{i}$ is a model and $\bar{a}_{i}$ is a finite sequence of elements in $M$ as follows:

1. Assume that $\left(N_{j}: j \leq i\right)$ and $\left(\bar{a}_{j}: j<i\right)$ have been defined. If there is $\bar{a}$ in $M$ such that $\operatorname{tp}\left(\bar{a} / N_{i}\right) \not \perp \varphi(\bar{x}, \bar{b})$ let $\bar{a}_{i}=\bar{a}$ and let $N_{i+1}=\operatorname{Pr}_{a}\left(N_{i} \cup \bar{a}_{i}\right)$.

2. When i is a limit and $\left(N_{j}: j<i\right)$ are defined, let $N_{i}=\bigcup_{j<i} N_{j}$.

3. If any of the cases above does not hold, we define $N_{i+1}=N_{i}$ and $\bar{a}_{i}=\bar{a}_{j}$ for any $j<i$.

Note that $\left|N_{0}\right| \leq \chi$, so $\left|N_{i}\right| \leq \chi$ for all $i<\chi^{+}$.

Claim 3.8 There is $\alpha<\chi^{+}$such that $N_{\alpha+i}=N_{\alpha}$ for all $i<\omega$ (this means the process above stabilizes in less than $\chi^{+}$steps).

Proof Assume, in order to get a contradiction, that the sequence $\left(N_{i}: i<\chi^{+}\right)$ is increasing. Let $S$ be the set of (limit) ordinals $i$ such that $\operatorname{cf}(i)=\kappa_{r}(T) . S$ is a stationary set of cardinality $\chi^{+}$. For each $i \in S$, let $\delta_{i}<i$ be such that $\operatorname{tp}\left(\bar{a}_{i} / N_{i}\right)$ is strongly based on $N_{\delta_{i}}$. By Födor, there is a stationary set $S^{*} \subseteq S$ for which $\delta_{i}=\delta^{*}$ for all $i \in S^{*}$. Since $|S|=\chi^{+}$, we also have $\left|S^{*}\right|=\chi^{+}$.

For every $i \in S^{*}$ we have $\bar{a}_{i} \downarrow_{N_{\delta^{*}}} N_{i}$, so whenever $i, j \in S^{*}$ and $j<i$, $\bar{a}_{i} \downarrow_{N_{\delta^{*}}} \bar{a}_{j}$ and the sequence $\left(\bar{a}_{i}: i \in S^{*}\right)$ is independent over $N_{\delta^{*}}$. As $\left|N_{\delta^{*}}\right| \leq \chi$, 
by Fact 2.4 there is $S^{* *} \subseteq S^{*}$ of cardinality $\chi^{+}$such that $I=\left(\bar{a}_{i}: i \in S^{* *}\right)$ is an indiscernible sequence over $N_{\delta^{*}}$. Thus $I \subseteq M$ is a Morley sequence of some type $q$ over $N_{\delta^{*}}, q \not \perp \varphi(x, \bar{b})$, and $|I|=\chi^{+}$. Since $I \subset \cup_{j<\delta} M_{j}$ and $\operatorname{cf}(\delta) \leq \chi$, there is $j<\delta$ such that $\left|I \cap M_{j}\right| \geq \chi^{+}$. Thus, by exchanging $I$ for a subsequence, we may assume that $I \subset M_{j}$. Let $I_{0} \subseteq I$ be countably infinite, so $I^{+}=I \backslash I_{0}$ a Morley sequence over $I_{0}$.

Let $q^{+}=A v\left(I^{+} / I_{0}\right)$. Then $q^{+}$is parallel to $q$ and therefore $q^{+} \not \perp \varphi(x, \bar{b})$. Since $M_{j}$ is $\kappa_{r}(T)$-saturated, there is $\bar{b}^{*} \in M_{j}$ such that $\operatorname{tp}\left(\bar{b}^{*} / I_{0}\right)=\operatorname{tp}\left(\bar{b} / I_{0}\right)$. In particular, $\bar{b}^{*} \models p$. Then $q^{+} \not \perp \varphi\left(x, \bar{b}^{*}\right)$; so by Fact 2.7 , part 1 , we get that $\operatorname{dim}\left(q^{+}, M_{j}\right) \leq\left|\varphi\left(M_{j}, \bar{b}^{*}\right)\right|$, so $\chi^{+} \leq\left|\varphi\left(M_{j}, \bar{b}^{*}\right)\right|$. This contradicts the fact that $M_{j}$ is $\varphi, p$-good in $\chi$.

Let $\alpha$ be as in the claim and let $N^{+}=N_{\alpha}$, so $N_{0} \preceq N^{+},\left|N^{+}\right| \leq \chi$, and $\operatorname{tp}\left(M / N^{+}\right) \perp \varphi(x, \bar{b})$. Define $M^{*}=P r_{a}\left(N^{+} \cup M\right)$.

Claim 3.9 $\varphi\left(N^{+}, \bar{b}\right)=\varphi\left(M^{*}, \bar{b}\right)$.

Proof Suppose not, so assume there is $\bar{d} \in \varphi\left(M^{*}, \bar{b}\right) \backslash \varphi\left(N^{+}, \bar{b}\right)$. Then $M \bigotimes_{N^{+}} \bar{d}$

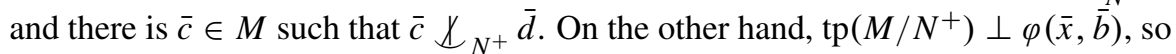
$\bar{c} \perp_{N^{+}} \bar{d}$, a contradiction.

Clearly, $\left|\varphi\left(N^{+}, \bar{b}\right)\right|=\left|\varphi\left(M^{*}, \bar{b}\right)\right| \leq \chi$ and $M \cup \bar{b} \subset M^{*}$ to finish the proof of (a).

Lemma 3.10 Assume $T$ is superstable and suppose that $M$ is an a-model. Then for any $\varphi(\bar{x}, \bar{a}) \in L(M)$ there exists a complete type $p_{M}$ over $M$ containing $\varphi(\bar{x}, \bar{a})$, which is orthogonal to all $\psi(\bar{y}, \bar{b}) \in L(M)$ with $C(\psi(\bar{y}, \bar{b}))<C(\varphi(\bar{x}, \bar{a}))$.

\section{Proof}

Claim 3.11 There is a finite set $A \subset M$ with $\bar{a} \subset A$ and a type $p_{A} \in S(A)$ such that

1. $C\left(p_{A}\right)=C(\varphi(\bar{x}, \bar{a}))$;

2. for any small $B \supset A$ (in $\mathbb{C}$ ) and forking extension $p_{B} \in S(B)$ of $p_{A}$, $C\left(p_{B}\right)<C\left(p_{A}\right)$.

Proof We define by induction an increasing chain of finite sets $\left(A_{i}: i<\omega\right)$ contained in $M$ and complete types $p_{i}$ over $A_{i}$ such that $p_{i+1}$ is an extension of $p_{i}$ and $C\left(p_{i}\right)=C(\varphi(\bar{x}, \bar{a}))$. For $i=0$, let $p_{0}$ be any extension of $\varphi(\bar{x}, \bar{a})$ to a complete type over a finite set $A_{0}$ with $\bar{a} \subset A_{0}$ and such that $C\left(p_{0}\right)=C(\varphi(\bar{x}, \bar{a}))$. The type $p_{0}$ exists by Definition 3.4, part 5 .

Assume we have defined $A_{i}$ and $p_{i}$. If there is a formula $\psi(\bar{x}, \bar{b}) \in L(M)$ with $C\left(p_{i} \cup \psi(\bar{x}, \bar{b})\right) \geq C\left(p_{i}\right)$ that forks over $A_{i}$, let $A_{i+1} \supset A_{i}$ be a finite subset of $M$ such that $\bar{b} \subset A_{i+1}$ and let $p_{i+1} \supset p_{i}$ be a complete type over $A_{i+1}$ containing $\psi(\bar{x}, \bar{b})$ satisfying $C\left(p_{i+1}\right)=C\left(p_{i}\right)$. Since $T$ is superstable this process stops in fewer than $\omega$ steps. Let $A=\cup A_{i}$, so $|A|<\omega$ and let $p_{A}=\cup p_{i}$.

Now let $B \subset \mathbb{C}$ be small; suppose that $A \subset B$ and that $p_{B} \in S(B)$ is a forking extension of $p_{A}$. Then there is $B_{0} \subset B$ finite with the same properties. Since $M$ is an $a$-model, we may assume $B_{0} \subset M$, so $C\left(p_{B_{0}}\right)<C\left(p_{A}\right)$. 
Claim 3.12 Let $B \supset A$ be small (subset of $\mathbb{C}$ ) and $p_{B} \in S(B)$ be a nonforking extension of $p_{A}$. Then $C\left(p_{B}\right)=C\left(p_{A}\right)$.

Proof Let $p_{B} \in S(B)$ be a nonforking extension of $p_{A}$. Let $N \supset A$ be a model such that $|N| \leq|T|$. By Definition 3.4, part 5, there is $p_{N} \supset p_{A}$ a complete type over $N$ such that $C\left(p_{N}\right)=C\left(p_{A}\right)$. By Claim 3.11, $p_{N}$ is a nonforking extension of $p_{A}$. Let $\Psi \in \operatorname{Aut}(\mathbb{C} / A)$ be such that $\Psi\left(p_{B}\right)$ is parallel to $p_{N}$; let $B^{\prime}=\Psi(B)$; and let $p_{B^{\prime}}=\Psi\left(p_{B}\right)$.

By Claim 3.11, all small types $q$ which are forking extensions of $p_{N}$ satisfy $C(q)<C\left(p_{N}\right)$. Since $p_{N}$ is stationary and $p_{N} \cup p_{B^{\prime}}$ is a nonforking extension of $p_{N}$, we must have $C\left(p_{N} \cup p_{B^{\prime}}\right)=C\left(p_{N}\right)$. Thus, $C\left(p_{B}\right)=C\left(p_{B^{\prime}}\right) \geq C\left(p_{N} \cup p_{B^{\prime}}\right)=$ $C\left(p_{N}\right)=C\left(p_{A}\right)$.

Let $e \in M$ realize $p_{A}$, let $A^{+}=A \cup\{e\}$, and let $p_{A}^{+}$be the nonforking extension of $\operatorname{stp}(e / A)$ to $A^{+}$. Then $p_{A}^{+}$is stationary and by the previous claim $C\left(p_{A}^{+}\right)=C\left(p_{A}\right)$. Let $p_{M}=\left.p_{A}^{+}\right|_{M}$ be the nonforking extension of $p_{A}^{+}$to $M$. Now we will show that $p_{M}$ satisfies the conclusion of the lemma.

Claim 3.13 $p_{M}$ is orthogonal to any $\psi(\bar{y}, \bar{b}) \in L(M)$ with $C(\psi(\bar{y}, \bar{b}))<$ $C(\varphi(\bar{x}, \bar{a}))$.

Suppose not. Then there is $\psi(\bar{y}, \bar{b}) \in L(M)$ such that $C(\psi(\bar{y}, \bar{b}))<C(\varphi(\bar{x}, \bar{a})))$ with $p_{M}$ nonorthogonal to $\psi(\bar{y}, \bar{b})$. Since $M$ is an $a$-model, there are $\bar{d} \models \psi(\bar{y}, \bar{b})$ and $\bar{c} \models p_{M}$ such that $\operatorname{tp}(\bar{c} / M \bar{d})$ forks over $M$. Let $\theta(\bar{x}, \bar{m}, \bar{d})$ be a formula in $\operatorname{tp}(\bar{c} / M \cup \bar{d})$ which forks over $M$, where $\bar{m} \subset M$. Let $D=A^{+} \cup \bar{m} \cup \bar{b}$ and let $p_{D}$ be an extension of $p_{A}^{+}$such that $C\left(p_{D}\right)=C\left(p_{A}^{+}\right)$. By Claim 3.11, $p_{D}$ is a nonforking extension of $p_{A}^{+}$. Thus $p_{D} \subset p_{M}$ and $\theta(\bar{x}, \bar{m}, \bar{d})$ is a formula in $\operatorname{tp}(\bar{c} / D \cup \bar{d})$ which forks over $D$.

Now let $E \in L(D)$ be the equivalence relation defined by

$$
E\left(\bar{x}_{1}, \bar{x}_{2}\right)=\forall \bar{y}\left(\psi(\bar{y}, \bar{b}) \Longrightarrow\left(\theta\left(\bar{x}_{1}, \bar{m}, \bar{y}\right) \Longleftrightarrow \theta\left(\bar{x}_{2}, \bar{m}, \bar{y}\right)\right)\right) .
$$

To finish the proof it suffices to show

1. there is $\bar{c}^{\prime \prime} \in \mathbb{C}$ such that $C\left(p_{D}(\bar{x}) \wedge E\left(\bar{x}, \bar{c}^{\prime \prime}\right)\right) \geq C\left(p_{D}(\bar{x})\right)$;

2. If $\bar{c}^{\prime \prime}$ is as in (1), then $E\left(\bar{x}, \bar{c}^{\prime \prime}\right) \wedge p_{D}(\bar{x})$ forks over $D$.

Then $p_{D} \supset p_{A}$ and $C\left(p_{D}\right)=C\left(p_{A}\right)$, so (1) and (2) contradict Claim 3.11.

We first show (2) assuming (1). Let $\bar{c}^{\prime \prime} \in \mathbb{C}$ be as in (1). Let $\bar{c}^{\prime} \in \mathbb{C}$ realize $p_{D}(\bar{x}) \wedge E\left(\bar{x}, \bar{c}^{\prime \prime}\right)$ and $\bar{d}^{\prime} \in \mathbb{C}$ be such that $\operatorname{tp}(\bar{c}, \bar{d} / D)=\operatorname{tp}\left(\bar{c}^{\prime}, \bar{d}^{\prime} / D\right)$. Since $\theta(\bar{x}, \bar{m}, \bar{d})$ forks over $D$, then $\theta\left(\bar{x}, \bar{m}, \bar{d}^{\prime}\right)$ forks over $D$. Notice that $p_{D}(\bar{x}) \wedge E\left(\bar{x}, \bar{c}^{\prime \prime}\right)$ implies $E\left(\bar{x}, \bar{c}^{\prime}\right)$ and thus also $\theta\left(\bar{x}, \bar{m}, \bar{d}^{\prime}\right)$. Thus $C\left(p_{D}(\bar{x}) \wedge E\left(\bar{x}, \bar{c}^{\prime \prime}\right)\right)$ forks over $D$.

Now we show (1). Assume, as a way to a contradiction, that for all $\bar{c}^{\prime \prime} \in \mathbb{C}$, $C\left(p_{D}(\bar{x}) \wedge E\left(\bar{x}, \bar{c}^{\prime \prime}\right)\right)<C\left(p_{D}\right)$. Since $\bar{c}^{\prime \prime} / E \in d c l^{\mathrm{eq}}(\psi(\bar{y}, \bar{b}), \bar{b}, \bar{m})$ by compactness there is a function $f(\bar{x}, \bar{b}, \bar{m})$ such that $\bar{c}^{\prime \prime} / E=f(\bar{e}, \bar{b}, \bar{m})$ for some $\bar{e} \models \psi(\bar{y}, \bar{b})$. By Definition 3.4, part 2, $C\left(\left\{\bar{c}^{\prime \prime} / E: \bar{c}^{\prime \prime} \in \mathbb{C}\right\}\right) \leq C(\psi(\bar{y}, \bar{b}))<C(\varphi(\bar{x}, \bar{a}))$ and by Definition 3.4, part 3, $C\left(p_{D}(x)\right)<C(\varphi(\bar{x}, \bar{a}))$, a contradiction.

Definition 3.14 Let $\varphi(\bar{x}, \bar{a}), \psi(\bar{y}, \bar{b}) \in L(\mathbb{C})$. We say that $\varphi(\bar{x}, \bar{a})$ is strongly foreign to $\psi(\bar{y}, \bar{b})$ and we write $\varphi(\bar{x}, \bar{a}) \perp{ }^{s t} \psi(\bar{y}, \bar{b})$ if, for all types $q(\bar{x})$ containing $\varphi(\bar{x}, \bar{a})$, we have $q(\bar{x}) \perp \psi(\bar{x}, \bar{a})$.

Finally we are ready to prove our main result: the bounds $C(\varphi(\bar{x}, \bar{a}))$ are optimal when $T$ is superstable. 
Theorem 3.15 Assume $T$ is superstable and let $M \models T$ be an a-model which is $W$-good. Let $h: L(\mathbb{C}) \rightarrow$ Car be such that $\sup \{h(\psi(\bar{y}, \bar{b})): \psi(\bar{y}, \bar{b}) \in L(\mathbb{C})$, $\left.C(\psi(\bar{y}, \bar{b})) \leq C(\varphi(\bar{x}, \bar{a})), \psi(\bar{y}, \bar{b}) \not^{\text {st }} \varphi(\bar{x}, \bar{a})\right\}<C(\varphi(\bar{x}, \bar{a}))$ for all $\varphi(\bar{x}, \bar{a})$ $\in L(\mathbb{C})$. Then there is an a-model $N \succeq M$ such that $N$ is $W$-good and $|\varphi(N, \bar{a})| \geq h(\varphi(\bar{x}, \bar{a}))$ for all $\varphi(\bar{x}, \bar{a}) \in L(N)$.

Proof For $\varphi(\bar{x}, \bar{a}) \in L(\mathbb{C})$, let $H(\varphi(\bar{x}, \bar{a}))=\sup \{h(\psi(\bar{y}, \bar{b})): \psi(\bar{y}, \bar{b}) \in L(\mathbb{C})$, $\left.C(\psi(\bar{y}, \bar{b})) \leq C(\varphi(\bar{x}, \bar{a})), \psi(\bar{y}, \bar{b}) \quad \not^{s t} \varphi(\bar{x}, \bar{a})\right\}$. Assume that $H(\varphi(\bar{x}, \bar{a}))<$ $C(\varphi(\bar{x}, \bar{a}))$ for all $\varphi(\bar{x}, \bar{a}) \in L(\mathbb{C})$. Given $M W$-good, we will show that there is $N$ an $a$-model such that $N \succeq M, N$ is $W$-good, and $|\varphi(N, \bar{a})| \geq H(\varphi(\bar{x}, \bar{a}))$ for all formulas $\varphi(\bar{x}, \bar{a}) \in L(N)$.

We construct inductively a chain of $a$-models $\left(M_{j}: j \leq \alpha\right)$ such that each $M_{j}$ is $W$-good and $M_{i} \preceq M_{j}$ for all $i<j$. Assume we have constructed $M_{i}$ for $i<j$.

Suppose that $j=i+1$ and that $\left|\varphi\left(M_{i}, \bar{a}\right)\right|<h(\varphi(\bar{x}, \bar{a}))$ for some formula $\varphi(\bar{x}, \bar{a}) \in L\left(M_{i}\right)$. By Lemma 3.10, there is $\bar{c} \in \mathbb{C} \backslash M_{i}$ realizing $\varphi(\bar{x}, \bar{a})$ and such that $\operatorname{tp}\left(\bar{c} / M_{i}\right) \perp \psi(\bar{y}, \bar{b})$ for all formulas $\psi(\bar{y}, \bar{b})$ with $C(\psi(\bar{y}, \bar{b}))<C(\varphi(\bar{x}, \bar{a}))$. Let $M_{i+1}=\operatorname{Pr}_{a}\left(M_{i} \cup \bar{c}\right)$. By Proposition 3.7, if $M_{i}$ is $W$-good, $M_{i+1}$ is also $W$ good. If there is no formula $\varphi(\bar{x}, \bar{a})$ as above, let $M_{i+1}=M_{i}$. If $j$ is a limit, let $M_{j}$ be $\cup_{i<j} M_{i}$.

Now we show this process ends. Let $\mu=2^{|M| \sup \{h(\psi) \mid \psi \in L\}}$.

Claim 3.16 The chain $\left(M_{i}: i<\mu^{+}\right)$stabilizes.

Proof Suppose the process continues through $\mu^{+}$steps. Let $S$ consist of the set of limit ordinals which are less than $\mu^{+}$. So $|S|=\mu^{+}$. If the chain does not stabilize, then for each $s \in S$ there is $i<s$ and a formula $\psi_{s}\left(\bar{y}, \bar{b}_{s}\right) \in L\left(M_{i}\right)$ such that $\left|\psi_{s}\left(M_{i}, \bar{b}_{s}\right)\right|<h\left(\psi_{s}\left(\bar{y}, \bar{b}_{s}\right)\right)$. By Födor's lemma, we can find $v<\mu^{+}$and $S^{*} \subset S$ such that $\left|S^{*}\right|=\mu^{+}$and $\psi_{s}\left(\bar{y}, \bar{b}_{s}\right) \in L\left(M_{\nu}\right)$ for all $s \in S^{*}$. Since $T$ is superstable, $\left|M_{i}\right| \leq \mu$ for each $i<\mu^{+}$, so $\left|M_{\nu}\right| \leq \mu$. Since $\left|S^{*}\right|=\mu^{+}$, there is a subset $S^{* *} \subset S^{*}$ such that $\left|S^{* *}\right|=\mu^{+}$and $\psi_{s}\left(\bar{y}, \bar{b}_{s}\right)=\psi(\bar{y}, \bar{b})$ for some formula $\psi(\bar{y}, \bar{b}) \in L\left(M_{\nu}\right)$. Thus we have increased $\psi(\bar{y}, \bar{b}) \mu^{+}$times, so there is $j<\mu^{+}$ such that $\left|\psi\left(M_{j}, \bar{b}\right)\right| \geq \mu$ and $\left|\psi\left(M_{j}, \bar{b}\right)\right| \geq h(\psi(\bar{y}, \bar{b}))$, a contradiction.

Let $k<\mu^{+}$be the first limit ordinal after which the chain $\left(M_{i}: i<\mu^{+}\right)$is constant.

Claim 3.17 The model $M_{k}=\cup_{i<k} M_{i}$ is W-good.

Proof We pointed out that if $M_{i}$ is $W$-good, then $M_{i+1}$ is also $W$-good. It remains to show that for limit ordinals $j \leq k, M_{j}$ is also $W$-good. So let $j \leq k$ be a limit ordinal and assume, as a way to a contradiction, that $M_{j}$ is not $W$-good. We may choose $j$ minimal with this property, so whenever $i<j, M_{i}$ is $W$-good. Since $M_{j}$ is not $W$-good, there is a triple $\left(\varphi(\bar{x}, \bar{y}), p(\bar{y}), \chi^{+}\right) \in W$ and $\bar{a} \in M_{j}$ a realization of $p$ such that $\left|\varphi\left(M_{j}, \bar{a}\right)\right| \geq \chi^{+}$. Now we divide the proof by cases.

Case 1 Suppose that $\operatorname{cf}(j)<\chi^{+}$. Since each $M_{i}$ is $W$-good for $i<j$, we have $\left|\varphi\left(M_{i}, \bar{a}\right)\right|<\chi^{+}$. Thus $\left|\varphi\left(\cup_{i<j} M_{i}, \bar{a}\right)\right|<\chi^{+}$and $\left|\varphi\left(M_{j}, \bar{a}\right)\right|<\chi^{+}$, so $M_{j}$ is $\varphi, p$-good, a contradiction.

Case 2 Suppose that $\operatorname{cf}(j) \geq \chi^{+}$. Since $\left|\varphi\left(M_{i}, \bar{a}\right)\right|<\chi^{+}$for each $i<j$, we can find a subsequence $\left(M_{i_{\alpha}}: \alpha<\chi^{+}\right)$of $\left(M_{i}: i<j\right)$ such that $\varphi\left(M_{i_{\alpha+1}}, \bar{a}\right) \supsetneq \varphi\left(M_{i_{\alpha}}, \bar{a}\right)$ whenever $\alpha<\chi^{+}$. So for each $\alpha<\chi^{+}$, there is a formula $\psi_{\alpha}\left(\bar{y}, \bar{b}_{\alpha}\right) \in L\left(M_{i_{\alpha}}\right)$ such that $h\left(\psi_{\alpha}\left(\bar{y}, \bar{b}_{\alpha}\right)\right)>\left|\psi_{\alpha}\left(M_{i_{\alpha}}, \bar{b}_{\alpha}\right)\right|$, and 
$\bar{c}_{\alpha+1} \in M_{i_{\alpha+1}} \backslash M_{i_{\alpha}}$ realizing $\psi_{\alpha}\left(\bar{y}, \bar{b}_{\alpha}\right)$ such that $\operatorname{tp}\left(\bar{c}_{\alpha+1} / M_{i_{\alpha}}\right) \not \perp \varphi(\bar{x}, \bar{a})$. Let

$\bar{d}_{\alpha+1} \in M_{i_{\alpha+1}}$ satisfy $\varphi(\bar{x}, \bar{a})$ such that $\bar{c}_{\alpha+1} \bigotimes_{M_{i_{\alpha}}} \bar{d}_{\alpha+1}$. Let $\theta\left(\bar{x}, \bar{m}_{i_{\alpha}}, \bar{c}_{\alpha+1}\right)$ be a formula in $\operatorname{tp}\left(\bar{d}_{\alpha+1} / M_{i_{\alpha}} \cup \bar{c}_{\alpha+1}\right)$ which forks over $M_{i_{\alpha}}$ and such that $\theta\left(\bar{y}, \bar{m}_{i_{\alpha}}, \bar{c}_{\alpha+1}\right)$ implies $\psi_{\alpha}\left(\bar{y}, \bar{b}_{\alpha}\right)$.

Let $E_{\alpha}$ be an equivalence relation as in Fact 2.8. Then $E_{\alpha}$ is over $\varphi\left(M_{i_{\alpha}}, \bar{a}\right) \cup \bar{a}$, $\left|\bar{d} / E_{\alpha}: \bar{d} \in M_{i_{\alpha}}\right| \leq\left|\psi_{\alpha}\left(M_{i_{\alpha}}, \bar{b}_{\alpha}\right)\right|$ and $\bar{d}_{\alpha+1} / E_{\alpha} \notin M_{i_{\alpha}}^{\mathrm{eq}}$.

Let $S$ consist of the limit ordinals which are less than $\chi^{+}$. For each $\alpha \in S$, there is $\gamma<\alpha$ such that $E_{\alpha}$ is over $\varphi\left(M_{i_{\gamma}}, \bar{a}\right) \cup \bar{a}$. By Födor's lemma, there is $\delta<\chi^{+}$and a subset $I$ of $S$ of cardinality $\chi^{+}$such that $E_{\alpha}$ is over $\varphi\left(M_{\delta}, \bar{a}\right) \cup \bar{a}$ for all $\alpha \in I$. By hypothesis $M_{\delta}$ is $W$-good, so $\left|\varphi\left(M_{\delta}, \bar{a}\right) \cup \bar{a}\right|<\chi^{+}$and there are less than $\chi^{+}$many definable equivalence relations over $\varphi\left(M_{\delta}, \bar{a}\right) \cup \bar{a}$. Thus, by reducing further the set $I$, we may assume that there is a single definable equivalence relation $E$ over $\varphi\left(M_{\delta}, \bar{a}\right) \cup \bar{a}$ such that $E_{\alpha}=E$ for all $\alpha \in I$. Since $\bar{d}_{\alpha+1} / E \notin M_{i_{\alpha}}^{\mathrm{eq}}$ for all $\alpha \in I$, the sequence $\left(\bar{d}_{\alpha+1} / E: \alpha \in I\right)$ is formed by distinct elements so $\left|\left(\bar{d}_{\alpha+1} / E: \alpha \in I\right)\right|=\chi^{+}$. This implies that for some $\alpha \in I$, $\left|\left(\bar{d}_{\gamma+1} / E: \gamma<\alpha\right)\right| \geq \chi$. Thus $\chi \leq\left|\bar{d} / E_{\alpha}: \bar{d} \in M_{i_{\alpha+1}}\right| \leq\left|\psi_{\alpha}\left(M_{i_{\alpha+1}}, \bar{b}_{\alpha}\right)\right|$. This implies $h\left(\psi_{\alpha}\left(\bar{x}, \bar{b}_{\alpha}\right)\right)>\chi$, so $H(\varphi(\bar{x}, \bar{a})) \geq \chi^{+}$, a contradiction.

\title{
References
}

[1] Buechler, S., Essential Stability Theory, Perspectives in Mathematical Logic. SpringerVerlag, Berlin, 1996. Zbl 0864.03025. MR 1416106. 344

[2] Pillay, A., Geometric Stability Theory, vol. 32 of Oxford Logic Guides, The Clarendon Press, New York, 1996. Zbl 0871.03023. MR 1429864. 344, 345

[3] Shelah, S., Classification Theory and the Number of Nonisomorphic Models, 2d edition, vol. 92 of Studies in Logic and the Foundations of Mathematics, North-Holland Publishing Co., Amsterdam, 1990. Zbl 0713.03013. MR 1083551. 343, 344, 345

\author{
Universidad Nacional de Colombia \\ Cra 30 nro 45-03 \\ Bogotá \\ COLOMBIA \\ ajberensteino@unal.edu.co \\ The Mathematical Research Institute \\ Tel Aviv University \\ ISRAEL \\ zshami@post.tau.ac.il
}

